\title{
Evaluation of Signal Features used for Impact Localization on a Center Console with Piezoceramic Sensors
}

\author{
André Böhle \\ Chemnitz University of Technology \\ Professorship of Computer Engineering \\ E-mail: $\underline{\text { andbo@hrz.tu-chemnitz.de }}$
}

\author{
René Schmidt \\ Chemnitz University of Technology \\ Professorship of Computer Engineering \\ E-mail: rene.schmidt@informatik.tu- \\ chemnitz.de
}

\author{
Wolfram Hardt \\ Chemnitz University of Technology \\ Professorship of Computer Engineering \\ E-mail: cera@cs.tu-chemnitz.de
}

\begin{abstract}
Smart components are increasingly of interest in research and industry due to their wide range of applications. An example of this is a current project of the Federal Excellence Cluster MERGE, which is concerned with the development of a center console that serves as a control element in an automobile and is executing actions by touching it. In order to facilitate this functionality, it is necessary to evaluate the electrical signals generated by piezoceramic sensors regarding to the localization of the impact. In this respect, various signal features are investigated for their suitability using a support vector machine. The results show that an impact localization can be realized by the energetic consideration of the signals but has limitations in the practical usability.
\end{abstract}

Keywords-impact localization, piezoceramic sensors, centre console, support vector machine, MERGE.

\section{INTRODUCTION}

The development of multifunctional lightweight components is becoming increasingly important, especially in the automotive industry. Through the low weight of the components reduces fuel consumption and emissions, while embedded systems are used to implement functions.

A current research project of the Federal Cluster of Excellence MERGE is the development of an intelligent input system in form of a center console for an automobile, which is shown in figure 1 [1]. The operation of the system is similar to that of a touch display, with the touch of a finger on the

\footnotetext{
${ }^{1}$ Copyright $@ 2019$ by ESS Journal
}

surface, which activates application-specific behaviour, such as opening windows or the trunk of a car [1]. For the implementation a signal processing system is required, which is able to determine the point of contact between finger and user interface and guarantees energy efficiency and real-time capability as an embedded system.

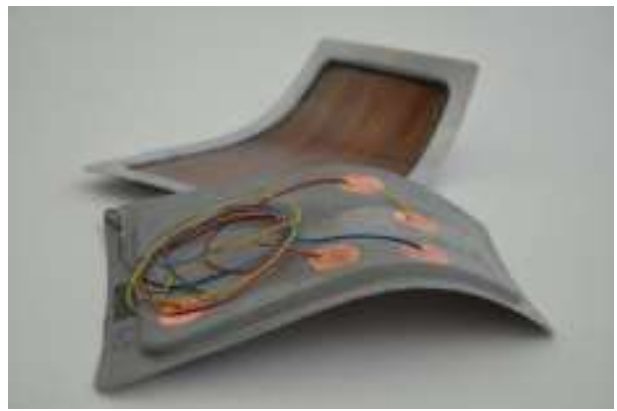

Figure 1: center console with piezoceramic sensors.

With the help of four piezoceramic sensors located on the center console, the mechanical pressure acting on the surface of the center console is converted into electrical analog signals [2]. These signals are digitized and then analyzed to find signal features that, in combination with a support-vector-machine, enable repeatable and intensity independent impact localization.

The paper is structured as follows. The next section considers the related work. That is followed by a short chapter on data acquisition. Afterwards chapter IV describes signal analysis and feature extraction. Finally, in section $\mathrm{V}$ the 
support vector machine is taught the signal features and the results are evaluated.

\section{RELATED WORK}

In this chapter different possibilities are considered which can be used for impact localization. Thereby the number and arrangement of the piezoceramic sensors has a decisive influence because they enable longitudinal and transversal impact localization. For this reason, three of the four sensors are arranged centrally in a row. The fourth sensor is located decentralized, to the side of the sensor row. This is a fact considered for all subsequent research.

The most common method to determine the position of impacts is the use of machine learning algorithms and neural networks [3]. This has the advantage that the concrete structure of the object does not have to be known and if there are changes in the structure, a new learning of the algorithm is sufficient [1]. Another option is to use a model of the object to calculate the potential position of the impact based on various input patterns [4]. Regardless of which of the methods is used, signal features are required as inputs for the algorithms that provide information about the impact.

In a previous work, Schmidt et al. [2] use time differences between the single sensors, which were determined by thresholding, and evaluates the kernels of a support vector machine. With this feature, accuracies of up to $84 \%$ are achieved to correctly detect the impact, however the intensity dependence is impractical for use as a center console [2]. Therefore, signal features that guarantee intensity independence are considered in the following.

The phase shift is a physical quantity that indicates the angle by which two waves are shifted in relation to each other [5]. When the impact occurs, a wave spreads in a circle over the surface of the centre console. The wave reaches the sensors at different times, which in turn means that the sensors measure different values at the same time. The resulting signals are similar and shifted to each other, since damping and possible interference occur under real conditions. The phase shift between the signals changes depending on the distance between the impact location and the sensors. This dependency allows conclusions to be drawn about the position of the impact and this feature to be used for localization.

Another feature is the cross correlation, which describes the similarity of two different signals [6]. Analogous to the phase shift, the wave created by the impact results in similar signals. Based on the similarity, the cross correlation function can be used to calculate the temporal shift of the signals, which corresponds to the propagation time between the two sensors [6]. Depending on the position of the sensors, the propagation time between them changes depending on the impact location. This context allows the cross correlation to be used for impact localization.

Finally, the possibility of detecting and locating impacts through the investigation of short-term energy is considered. It is exploited that the energy added to the system by the impact is transported from the resulting wave to the sensors. The amplitude of the generated signal reflects this energy. In general, the more energy added to the system, the greater the amplitude of the signal. From the signals, the mean time and the mean frequency can be calculated, which provide a measure of the time or frequency at which the signal energy is concentrated [7]. These differ for the four sensors, which is why the impact location can be deduced from the tuples of the mean times and frequencies.

\section{DATA ACQUISITION}

To record the measured values, it must be ensured that the data is recorded at known points in time and that the time dependency of the sensors on each other is therefore given. For this reason, the Digilent Zybo Board is used for sampling, which has a Zynq-7000 System-on-Chip architecture that combines an ARM processor with an FPGA. This has the advantage that the processor can compute complex algorithms with low resource utilization, while the FPGA requires more resources as the computations become more complex but delivers a reliable result in a given time. The trade off between the performance of the hardware implementation and the flexibility of the software implementation makes it possible to meet the requirements of the signal processing system. The data transfer between FPGA and processor is done via an AXI interface, which provides fast and flexible communication methods with a high data throughput. This system also offers a good basis for the final implementation due to the guaranteed energy efficiency and the assurance of real-time capability. Furthermore, the same measurement method can be used to ensure reproducibility in the final implementation.

The evaluation data is generated by tapping the center console surface with the finger. In order to be able to assign the impacts correctly, the surface of the centre console was divided into 20 fields, whereby for the measured value recordings the centre of the respective field was always typed. When the finger hits the center console, a wave spreads over it, causing it to vibrate. As soon as the wave reaches the piezoceramic sensors, the piezo crystals undergo a structural change and thus generate a voltage [8]. The resulting analog signal is then converted into a digital signal by the integrated analog-todigital converter from XILINX. This so-called XADC is a multichannel analog-to-digital converter that samples the analog signals of the four sensors with a sample rate of 500 ksps. This results in the size of the buffer in which the values are temporarily stored after digitization. The buffer is realized as AXI Data FIFO, that summarizes the values in blocks, whereby the size of the blocks is configurable. Finally, the blocks are written to the on-chip memory, which the processor accesses after the measurement has been completed, and a CSV file is created from the digital values stored there. The data of the CSV file are processed further with the help of Matlab and form the basis for the analysis of the signal features to determine the impact position. 


\section{Signal ANALYSIS}

The impact localization is achieved by using a support vector machine, which is trained to the signal features. For this reason, it is important that the features form clusters, as these are used to determine the impact position. A high repeat accuracy is essential for reliable localization. In order to obtain a sufficiently large database for the analysis, 30 measured values were recorded for each field. When recording the measured values, an attempt was made to hit the same spot in the centres of the fields as far as possible, so that the area in which the impacts took place is as small as possible. Nevertheless, there are variances in the impact location and the intensity of the impact. The signals generated in this way correspond to the intended purpose and form a solid basis for the analysis of the signal features.

\section{A. phase difference}

First, the phase difference is considered, for the calculation of which the phases of two signals are subtracted from each other, whereby the phase of the signal from sensor 1 always serves as minuend. Since the phases of the signals are equivalent in the time domain and frequency domain, they are analyzed in the frequency domain, due to the better representation possibility. The Fast Fourier Transform is used to transfer signals from the time domain to the frequency domain. Through the complex representation, it is now possible to determine the phase as the angle between the imaginary part and the real part. However, the Fast Fourier Transform assumes that the signal can be continued periodically. Since this is not the case, all signals must first be weighted with a window function.

The expectation that the phase within a signal changes in time in the same way, resulting in a constant phase difference between two signals each, was not fulfilled. Instead, the phase difference change within the signal path can be described by a global/local minimum directly followed by a global/local maximum, as shown in figure 2. Due to the changes in the phase differences, it is no longer possible to assign the impact location unambiguously, which means that impact localization

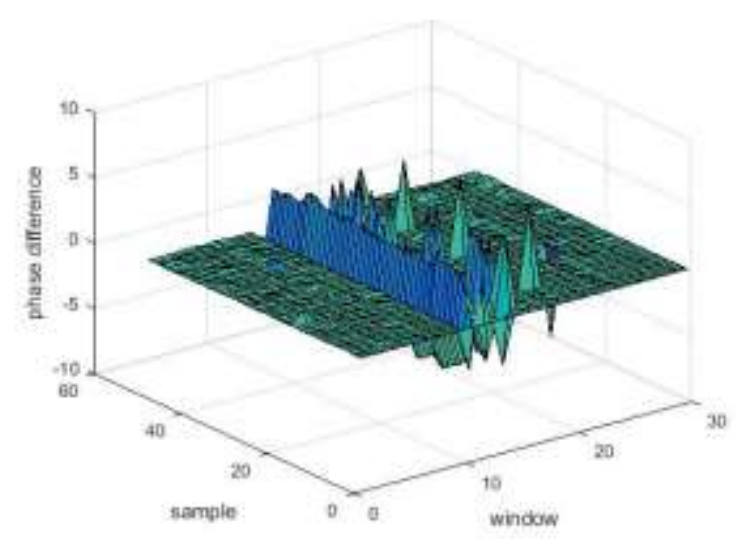

Figure 2: phase difference of a single measurement. cannot be realized with this feature. However, the phase shift pattern can be used to limit the signals to the portions containing the information regarding the impact, since the phase shift pattern always occurs when the impact is detectable in the signal. To limit the signal to the meaningful windows, a trigger is calculated. For this purpose, the mean value of the gradients of the phase differences per window is calculated in order to be able to determine the gradient again. The point at which the minimum occurs corresponds to the index value of the window with the most information about the impact. Even if the phase difference is not suitable for impact localization, it can be used in the analysis of the following signal features by limiting the number of windows to be viewed with the trigger found.

\section{B. cross-correlation}

The cross-correlation is the next signal feature to be investigated that is used to determine the point in time at which two signals are most similar. Analogous to the phase difference, in this case the signal of sensor 1 is also selected as reference point and the correlation between this and the other three signals is calculated. For the calculations, the functions "xcorr" and "normxcorr2" provided in Matlab were used, which return the correlation values and normalized correlation values as results. The positions of the maxima in the correlation functions correspond to the time at which the signals are most similar. When looking at them, it is noticeable that the maxima are all at zero time. Furthermore, the use of other signals instead of the signal of sensor 1 as a reference point has no influence on the results of the cross correlation. Since all correlation functions assume the largest value at time zero, a formation of clusters is not possible and the impact localization cannot be realized by calculating the temporal shift of the signals to each other.

\section{C. energy density}

Finally, the energetic investigation of the signals takes place. The signal energy is reflected in the square of the absolute value of the amplitude and changes depending on the intensity of the impact. Since the goal is to find intensity independent features for the impact localization, standardizations for the energy calculations will be carried out in the following or the ratio of the results will be formed in order to guarantee intensity independence.

First, the mean time is considered, which provides the point of time at which the signal energy is concentrated. The mean time is calculated using formula 1 for each signal from the centre console.

$$
t=\frac{1}{E} \sum_{i=1}^{N} t|x(i)|^{2}
$$


Basically, the four results obtained are sufficient for each experiment and for all fields to generate a data set for training the support vector machine and to test the mean time for its suitability for impact localisation. However, at this point the ratio of the mean times of the other three signals to signal 1 is formed in order to achieve better comparability.

The next feature to be considered is the mean frequency, which is a measure of the frequency by which the signal energy is concentrated. The equation 2 is used for its calculation.

$$
f=\frac{1}{E} \sum_{j=1}^{N} f|x(j)|^{2}
$$

For this purpose, the signals are windowed with a subsequent Fast Fourier Transform. Analogous to the mean time, the

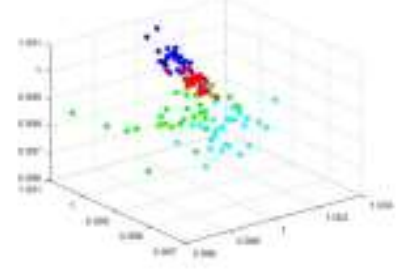

(a) ratios of the mean times

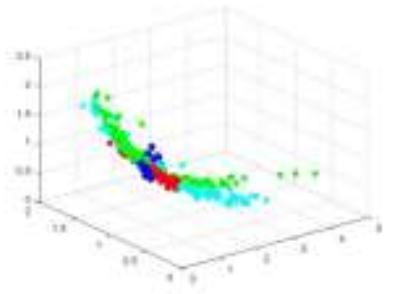

(b) ratios of the mean frequencies
Figure 3: ratios of mean time and mean frequency for four fields of the center console.

ratios of the mean frequencies are also formed here, which are used to train the support vector machine. Figure 3.a and 3.b, in which the resulting clusters for four fields of the center console are shown, illustrate once again that the mean time and the mean frequency are suitable features for training the support vector machine for impact localization.

Finally, the ratios of the signal energies of the windows, which were determined with the help of the trigger over the phase differences, are analysed. The index windows represent the energy of the impact, which differs depending on the impact location between the signals of the four sensors. With the calculation of the ratios of the signal energies, an intensityindependent consideration is made possible. As already described, the index windows for the calculations are determined via the phase shift trigger. The energy is then determined using these windows by adding up the square of the absolute value of the individual values. At the end the relations between the energies are calculated, whereby the energy from the index window of the signal of sensor 1 serves again as reference point. For the ratios of the signal energies of the index windows in the time domain, as well as in the frequency domain, cluster formations are recognizable, which were illustrated by four fields of the center console in figures 4.a and 4.b. Therefore two further features have been found, which are suitable for training the support vector machine.

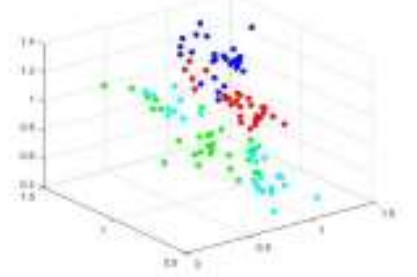

(a) ratios of energies in the time domain

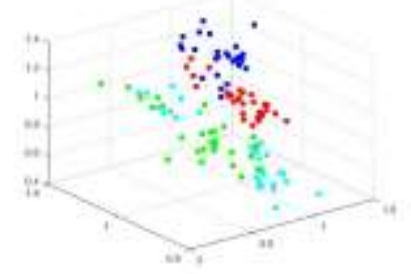

(b) ratios of energies in the frequency domain
Figure 4: ratios of energies for four fields of the center console.

\section{RESULTS}

In this chapter, the evaluation of the cluster forming signal features is performed using the support vector machine from the "LIBSVM" [9] library to evaluate their accuracy in impact localization. First, the use of the signal features for different kernel types of the support vector machine is investigated. These are the polynomial kernel, the radial kernel and the sigmoid kernel. In addition, the "LIBSVM" [9] library offers a scaling function that allows the training and test data to be scaled to any value range. In this case, scaling to the value range from -1 to 1 takes place. In order to be able to assess the influence of scaling, each signal characteristic is considered with and without scaling. The results of the calculations for the respective signal features of all tests for all fields of the centre console serve as test data for training the support vector machine.

TABLE I

\section{RESULTS OF THE SUPPORT VECTOR MACHINE REGARDING} DIFFERENT KERNELS

\begin{tabular}{|c|c|c|c|c|}
\hline Signal feature & Scaling & $\begin{array}{c}\text { Accuracy } \\
\text { (polynomial } \\
\text { kernel) }\end{array}$ & $\begin{array}{c}\text { Accuracy } \\
\text { (radial } \\
\text { kernel) }\end{array}$ & $\begin{array}{c}\text { Accuracy } \\
\text { (sigmoid } \\
\text { kernel) }\end{array}$ \\
\hline $\begin{array}{c}\text { Ratio of mean } \\
\text { time }\end{array}$ & + & $43.0 \%$ & $63.4 \%$ & $48.2 \%$ \\
\cline { 2 - 5 } & - & $25.0 \%$ & $29.6 \%$ & $19.9 \%$ \\
\hline $\begin{array}{c}\text { Ratio of the mean } \\
\text { frequency }\end{array}$ & + & $19.8 \%$ & $21.1 \%$ & $11.8 \%$ \\
\cline { 2 - 5 } & - & $27.2 \%$ & $35.4 \%$ & $9.7 \%$ \\
\hline $\begin{array}{c}\text { Ratio of the } \\
\text { energy of the } \\
\text { index windows in } \\
\text { the time domain }\end{array}$ & - & $68.8 \%$ & $65 \%$ & $61.6 \%$ \\
\cline { 2 - 5 } & + & $65.1 \%$ & $68.7 \%$ & $61.0 \%$ \\
\hline $\begin{array}{c}\text { Ratio of the } \\
\text { spectral energy of } \\
\text { the index } \\
\text { windows }\end{array}$ & + & $70.2 \%$ & $72.4 \%$ & $46.1 \%$ \\
\cline { 2 - 5 } & - & $6.2 \%$ & 61.0 \\
\hline
\end{tabular}

The results obtained are shown in table 1. This shows that the sigmoid kernel is by far the worst performer and provides the lowest accuracy of all tested signal features. On the other hand, the generated accuracies of the polynomial kernel and the 
radial kernel are approximately equal, whereby the radial kernel always has the higher accuracy of both. The scaling of the data results in a deterioration of $5 \%$ to $10 \%$ of the accuracy of all signal properties, except for the ratio of the mean time, for the polynomial kernel and the radial kernel. The sigmoid kernel benefits from the scaling of the values with a significant improvement in the accuracy of all signal features, but its generated accuracy is still much lower than that of the other two kernels. The best results with an accuracy of approximately $70 \%$ are provided by the ratios of the energies of the index windows in the time domain and frequency domain. Followed by the mean time ratios using scaling and the radial kernel with an accuracy of $63 \%$. These three signal features generated distinguishable clusters with a low dispersion during their analysis and thus achieved the highest accuracies. For the calculation of the mean frequencies, it turns out that they are not suitable for impact localization with an accuracy of only $20 \%$. This can be explained by the strong overlap of the clusters, which means that when subdividing the data in the support vector machine, parts of the clusters were assigned to other classes.

In overall terms, the results are not satisfactory. Based on the intended use of the centre console as a control unit in an automobile, it must be regarded as a safety-critical system, which is why a probability of success of $70 \%$ does not meet the requirements. In order to do justice to the use of the centre console, accuracies of $90 \%$ and more are desirable. An improvement can be achieved by looking at a smaller number of fields on the center console.

The following investigation compares the use of the signal features of four fields and all fields of the center console. The four fields considered are the fields of the first row of the centre console. For the calculation, the support vector machine is used with the radial kernel, as this provides the best results and the calculated signal features of all experiments serve as training data and test data. The result is shown in table 2 .

TABLE II

\section{RESULTS OF THE SUPPORT VECTOR MACHINE REGARDING DIFFERENT FIELDS OF THE CENTER CONSOLE}

\begin{tabular}{|c|c|c|c|}
\hline Signal feature & Scaling & $\begin{array}{c}\text { Accuracy } \\
\text { (4 fields) }\end{array}$ & $\begin{array}{c}\text { Accuracy } \\
\text { (all fields) }\end{array}$ \\
\hline Ratio of mean time & + & $93.0 \%$ & $63.4 \%$ \\
\cline { 2 - 4 } & - & $72.4 \%$ & $29.6 \%$ \\
\hline Ratio of the mean frequency & + & $64.3 \%$ & $21.1 \%$ \\
\cline { 2 - 4 } & - & $64.6 \%$ & $35.4 \%$ \\
\hline $\begin{array}{c}\text { Ratio of the energy of the } \\
\text { index windows in the time } \\
\text { domain }\end{array}$ & + & $91.0 \%$ & $65.8 \%$ \\
\cline { 2 - 4 } & - & $88.3 \%$ & $69.4 \%$ \\
\hline $\begin{array}{c}\text { Ratio of the spectral energy } \\
\text { of the index windows }\end{array}$ & + & $91.0 \%$ & $68.7 \%$ \\
\cline { 2 - 4 } & - & $90.0 \%$ & $72.4 \%$ \\
\hline
\end{tabular}

According to the initial assertion, table 2 shows that the accuracy of the signal features is significantly higher for only four considered fields than for all fields. In addition, the ratios of the energies of the index windows, as well as the ratio of the mean time reach an accuracy of over $90 \%$. This shows, on the one hand, that the three signal properties are suitable for impact localization, but their accuracies strongly depend on the number of fields observed, and on the other hand, that transverse impact localization is possible because the four fields examined are horizontally in a row and the sensor row is perpendicular to these. In this case, even the scaling of these three signal features results in a slight improvement in accuracy. The research shows that impact localization is possible with the ratios of the energies of the index windows and the ratio of the mean time of the signals, but their practical use depends strongly on the number of fields used.

\section{FUTURE WORK}

The results of the research show that it is possible to localize the impacts for a small number of fields of the center console with a high probability by energetic observation of the signals. However, the accuracy decreases as more fields are included in the calculations. In this respect, in order to improve the results, impact localisation could be implemented in several stages. Therefore several fields of the center console are combined to a new, larger field. Then the impact is first assigned to a large field and then another calculation, which considers the subfields of the large field, is used to assign the impact to the original field. The advantage of this is that fewer clusters are used for the respective calculations, which means that there are less overlaps. However, this goes hand in hand with a trade off between usage and resource utilization, because depending on the number of levels at least two support vector machines have to be trained with regard to different data sets.

With an accuracy of $70 \%$, the energy ratios of the index windows already provide a good basis for impact localization. This accuracy was achieved using a support vector machine, which was trained on the data of 30 impacts per field of the center console. The size of the data set is decisive for the creation of the support vector machine classes to which the test data is assigned. Since the training data is a relatively small amount of data, the same research should be repeated with a larger data set to provide more accurate information about the detection rate.

With the listed results a basis for the impact localisation concerning the centre console is created, but there is also room for further investigations, which was clarified by the possibilities just described.

\section{ACKNOWLEDGMENT}

We gratefully acknowledge the cooperation of our project partners and the financial support of the DFG (Deutsche Forschungsgemeinschaft) within the Federal Cluster of Excellence EXC 1075 MERGE. 


\section{REFERENCES}

[1] Ullmann, Frank, Wolfram Hardt, and Vadim Zhmud. "Machine learning algorithms for impact localization on formed piezo metal composites." 2017 International Siberian Conference on Control and Communications (SIBCON). IEEE, 2017.

[2] Schmidt, René, et al. "Hybrid Laminate for Haptic Input Device with Integrated Signal Processing." Applied Sciences 8.8 (2018): 1261.

[3] Worden, Keith, and Janice M. Dulieu-Barton. "An overview of intelligent fault detection in systems and structures." Structural Health Monitoring 3.1 (2004): 85-98.

[4] Tracy, Michael, and Fu-Kuo Chang. "Identifying impacts in composite plates with piezoelectric strain sensors, part I: Theory." Journal of Intelligent Material Systems and Structures 9.11 (1998): 920-928.

[5] Hering, Ekbert, Rolf Martin, and Martin Stohrer. "Physik für Ingenieure.” Springer-Verlag, 2007.
[6] Hoffmann, Rüdiger, and Matthias Wolff. "Intelligente Signalverarbeitung 1: Signalanalyse." Vol. 1. Springer-Verlag, 2014.

[7] Puente León, Fernando, and Sebastian Bauer. " Praxis der Digitalen Signalverarbeitung." 2. überarb. KIT Scientific Publishing, 2017.

[8] Melz, Tobias. "Entwicklung und Qualifikation modularer Satellitensysteme zur adaptiven Vibrationskompensation an mechanischen Kryokühlern." Diss. Technische Universität, 2002.

[9] Chang, Chih-Chung, and Chih-Jen Lin. "LIBSVM: A library for support vector machines." ACM transactions on intelligent systems and technology (TIST) 2.3 (2011): 27. 